\title{
Analytical Investigation of the Single-Particle Energy Spectrum in Magic Nuclei of ${ }^{56} \mathrm{Ni}$ and ${ }^{116} \mathrm{Sn}$
}

\author{
J. A. Obu, E. S. William, I. O. Akpan, E. A. Thompson and E. P. Inyang
}

\section{ABSTRACT}

\begin{abstract}
The analytical solutions of the radial D-dimensional Schrödinger equation for the Yukawa potential plus spin-orbit and Coulomb interaction terms are presented within the framework of the Nikiforov-Uvarov method by using the Greene-Aldrich approximation scheme to the centrifugal barrier. The energy eigenvalues obtained are employed to calculate the singleenergy spectrum of ${ }^{56} \mathrm{Ni}$ and ${ }^{116} \mathrm{Sn}$ for distinct quantum states. We have also obtained corresponding normalized wave functions for the magic nuclei manifested in terms of Jacobi polynomials. However, the energy spectrum without Spin-orbit and Coulomb interaction terms precisely matches the quantum mechanical system of the Yukawa potential field at any arbitrary state.
\end{abstract}

Keywords: D-dimensional Schrodinger equation, Nikiforov-Uvarov method, spin-orbit and Coulomb interaction terms, Yukawa potential.
Published Online: December 8, 2020

ISSN: $2684-4451$

DOI : $10.24018 /$ ejphysics.2020.2.6.28

\section{J. A. Obu}

University of Calabar, Calabar, Nigeria. (e-mail: abebeobu@gmail.com)

E. S. William*

University of Calabar, Calabar, Nigeria. (e-mail: williameddyphysics@gmail.com) I. O. Akpan

University of Calabar, Calabar, Nigeria.

(e-mail: itayenakpan@yahoo.com)

E. A. Thompson

University of Calabar, Calabar, Nigeria.

(e-mail: edyy7000@yahoo.com)

E. P. Inyang

University of Calabar, Calabar, Nigeria (e-mail: etidophysics@gmail.com)

*Corresponding Author

\section{INTRODUCTION}

The ideal description and interpretation of the features of a many-body system can be studied considering the atomic nucleus as intricate quantum system designed by two distinct fermions, the proton, and the neutron [1], [2]. After the discovery of the atomic nucleus, its structure has been studied in detail using different nuclear models. The nuclear shell model (arrangement of protons and neutrons within a nucleus) led nuclear research to the modern model of the atomic nucleus [3], [4]. The strength of the nuclear shell model is well established for nuclei approaching the valley of stability. It effectively predicts and explains the occurrence of magic numbers [5]-[8]. Magic numbers corresponding to the filling of nuclear shells of singleparticle levels exist since the middle of the past century [9][13]. It can be explained in terms of the shell model of the nucleus. This model uses quantum mechanics to describe the energetics of the nucleons [14] by assuming that each nucleon moves independently in the nucleus not influenced by the motion of the other nucleons. Furthermore, each nucleon moves in a potential well, which is constant from the center of the nucleus to its edge, where it increases rapidly by its energy.

The interaction between a proton and a neutron with all other nucleons generates an average potential field where each nucleon moves. The interacted nucleons have a nuclear mean-field potential that depends on the position coordinates [15]. There is a capability to calculate the mean- field potential in nuclei. This purpose ranged from the empirical picture to fit the experimental data and to derive it on a small scale from the bare nucleon-nucleon potential. Thus, there is no unique nucleon-nucleon potential to be the start point [16], [17]. However, since the strong nuclear force is short ranged, we would expect the potential to follow the form of the density distribution of nucleons in the nucleus. Therefore, any short-range potential that would reproduce a single-particle energy spectrum must be constrained to this shape.

In quantum mechanics, Schrodinger equation (SE) is one of the central equations which draw great attention in physical sciences. Much advanced mathematical methods with specific potential have been used to solve this equation. Among the most popular methods are Nikiforov-Uvarov method NU [18]-[24], asymptotic iteration method [25][28], supersymmetric shape invariance approach [29]-[31], factorization method [32]. Studies show that the extension of physical problems to higher-dimensional space is of great concern [33]. Also, [34] noted that the exact solutions of both the relativistic and nonrelativistic wave equation with certain physical potential in higher dimensions are remarkably important not only in physics and chemistry but also in pure and applied mathematics.

More so, a single potential alone cannot reproduce experimentally observed magic numbers. Including the spinorbit and Coulomb interaction terms to the mean-field potential, it is possible to reproduce these numbers [35][37]. The effect of spin-orbit coupling in the mean-field 
arises if there is a contribution of orbits with $l \geq 0$. Such trajectories occur when the constraint on the particle reflects the nuclear radius [38]. The contribution of the Coulomb term is as a result of interactions between the protons in the nucleus.

Hence, this study aims to investigate the analytical solution of the radial D-dimensional Schrodinger equation for the Yukawa potential in the presence of spin-orbit and Coulomb interaction terms to study magic nuclides of ${ }^{58} \mathrm{Ni}$ and ${ }^{116} \mathrm{Sn}$. The Yukawa potential [39] is a useful nonrelativistic potential describing the strong interactions between nucleons [40], [41]. The potential is of the form:

$$
V_{Y}(r)=-\frac{\eta e^{-\zeta r}}{r}
$$

where $\eta$ is the strength of the Yukawa potential, $\zeta$ is the screening parameter and $r$ is the nuclear distance between two interacting nucleons [42]. In quantum mechanics, the physics of this potential depends strongly on the value of the screening parameter and also on the type of the problem under consideration.

\section{REVIEW OF NIKIFOROV-UVAROV (NU) METHOD}

We briefly introduce the NU method in this section; the details can be found in ref. [18]. This method is based on solving the second-order differential equation of the hypergeometric type:

$$
\psi^{\prime \prime}(z)+\frac{\tilde{\tau}(z)}{\sigma(z)} \psi^{\prime}(z)+\frac{\tilde{\sigma}(z)}{\sigma^{2}(z)} \psi(z)=0
$$

where $\sigma(z)$ and $\tilde{\sigma}(z)$ are polynomials at most seconddegree, $\tilde{\tau}(z)$ is a first-degree polynomial [43]. The particular solution of (2) is found by decomposing the wave function $\psi(z)$ as:

$$
\psi(z)=\phi(z) y(z)
$$

which result in a hypergeometric-type equation of the form:

$$
\sigma(z) y^{\prime \prime}(z)+\tau(z) y^{\prime}(z)+\lambda y(z)=0
$$

The first part of (3), $\phi(z)$ is the solution of the differential equation:

$$
\sigma(z) \phi^{\prime}(z)-\pi(z) \phi(z)=0
$$

where

$$
\tau(z)=\tilde{\tau}(z)+2 \pi(z)
$$

and $\lambda$ in (4) is define as:

$$
\lambda=\lambda_{n}=-n \tau^{\prime}(s)+\frac{n(n-1)}{2} \sigma^{\prime \prime}(s)=0,(n=0,1,2, \ldots)
$$

The $\tau(z)$ is a polynomial and the first derivative $\tau^{\prime}(z)$ must be negative for proper solutions. The second part in (3) is a hypergeometric-type function obtained by Rodrigues relation:

$$
y_{n}(z)=\frac{B_{n}(z)}{\rho(z)} \frac{d^{n}}{d z^{n}}\left[\sigma^{n}(z) \rho(z)\right]
$$

We define $B_{n}$ as a constant related to normalization and $\rho(z)$ is of the form:

$$
\frac{d}{d z}[\sigma(z) \rho(z)]=\tau(z) \rho(z)
$$

We also define the function $\pi(z)$ and the parameter $\lambda$ as:

$$
\pi(z)=\frac{\sigma^{\prime}(z)-\tilde{\tau}(z)}{2} \pm \sqrt{\left(\frac{\sigma^{\prime}(z)-\tilde{\tau}(z)}{2}\right)^{2}-\tilde{\sigma}(z)+k \sigma(z)}
$$

Where $\pi(z)$, is a polynomial of first degree, and

$$
\lambda=k+\pi^{\prime}(z)
$$

To obtain the value of $\mathrm{k}$, the term under the square root sign in (10) is set to zero [43]. Subsequently, by solving (7) and (11), we obtained the energy eigenvalue equation.

\section{SOLUTION OF THE SCHRODINGER EQUATION WITH YuKaWA POTENTIAL Plus SPIN-ORBIT AND COULOMB INTERACTION TERMS}

The Schrodinger equation in D - dimensionional space of nucleons which interacts with each other is of the form [43]:

$\frac{\hbar^{2}}{2 \mu}\left[\frac{d^{2}}{d r^{2}}+\frac{D-1}{r} \frac{d}{d r}-\frac{\ell(\ell+D-2)}{r^{2}}\right] R_{n \ell j}(r)=\left(E_{n \ell j}-V(r)\right) R_{n j j}(r)$

where $D=3 N-3$. Here, $D$ is the space dimension, $N$ is the number of particles, $\mu$ is the reduced mass of a single nucleus, $E_{n l j}$ is the energy spectra, $\hbar$ is the reduced Planck's constant and $n, \ell$ and $j$ are the principal, orbital and total angular momentum quantum numbers respectively. By using of the change of variable $\varphi_{n l j}(r)=r R_{n \ell j}(r)$, we have:

$\varphi_{n l j}(r)=R_{n \ell j}(r)+r \frac{R_{n \ell j}(r)}{d r}$.

For two particles system (i. e. proton and neutron where $N=2$ and $D=3$ ), (12) reduces to: 


$$
\frac{d^{2} \varphi_{n \ell j}(r)}{d r^{2}}+\frac{2 \mu}{\hbar^{2}}\left[E_{n \ell j}-V(r)-\frac{\hbar^{2} \ell(\ell+1)}{2 \mu r^{2}}\right] \varphi_{n \ell j}(r)=0,
$$

The spin-orbit term of the central Yukawa potential is of the form:

$V(r)_{(s 0)}(r)=\frac{1}{2} \eta_{(s 0)}\left(\frac{r_{0}}{\hbar}\right)^{2} \frac{1}{r}\left[\frac{d}{d r} \frac{e^{-\zeta r}}{r}\right]<\vec{\ell} \cdot \vec{s}>$

where the spin-orbit coupling constant, $\eta_{\left(s_{0}\right)}=0.44 \eta$. For spin half particle, the spin-orbit coupling term, $<\vec{\ell} \cdot \vec{s}>=\frac{1}{2} \hbar^{2}\left(j(j+1)-l(l+1)-\frac{3}{4}\right)$. In addition to the mean-field plus spin-orbit interaction, protons in nuclei interact together via the Coulomb force, which is defined by the following relation, considering nuclei as a sphere with a constant charge density [44]:

$$
V_{c}(r)= \begin{cases}\frac{e^{2}}{R_{c}}\left[3-\left(\frac{r}{R_{c}}\right)^{2}\right] & r \leq R_{c}, \\ \frac{8 e^{2}}{r}, & r \geq R_{c},\end{cases}
$$

the effective potential is given as the combination of (1), (15) and (16) for $r<R_{c}$ :

$V(r)=-\frac{\eta e^{-\zeta r}}{r}+\frac{1}{2} \eta_{(s o)} r_{0}^{2} \frac{1}{r}\left[\frac{d}{d r} \frac{e^{-\zeta r}}{r}\right]\left(j(j+1)-l(l+1)-\frac{3}{4}\right)+$ $+\left(\frac{3 e^{2}}{\pi \varepsilon_{0} R_{c}}-\frac{e^{2} r^{2}}{\pi \varepsilon_{0} R_{c}^{3}}\right)$

where $R_{c}$ is the Coulomb radius, $e$ is the electronic charge and $\varepsilon_{0}$ is the medium of the particles. By substituting (17) into (14), we have: $\frac{1}{r^{2}} \approx \frac{\zeta^{2}}{\left(1-e^{-\zeta r}\right)^{2}}$

By using (19), (20), and the change of variable in (19), the Schrodinger equation reads

$\frac{d^{2} \varphi_{n l j}(z)}{d z}+\frac{1}{z} \frac{d R(z)}{d z}+\frac{2 \mu}{\zeta^{2} z^{2} \hbar^{2}}\left[\begin{array}{l}E_{n j}+\frac{\eta \zeta z}{(1-z)}+\frac{1}{2} \eta_{(s o)} r_{0}^{2} \zeta^{3}\left(\frac{z}{(1-z)^{2}}+\frac{z}{(1-z)^{3}}\right) \\ \left(j(j+1)-l(l+1)-\frac{3}{4}\right)-\left(\frac{3 e^{2}}{\pi \varepsilon_{0} R_{c}}-\frac{e^{2} \frac{(1-z)^{2}}{\zeta^{2}}}{\pi \varepsilon_{0} R_{c}^{3}}\right) \\ -\frac{\hbar^{2}}{2 \mu} \frac{\zeta^{2}}{(1-z)^{2}} l(l+1)\end{array}\right] \varphi_{n l j}(z)=0$

To reach an approximate solution in (21), we apply the principle of [48] to higher degree polynomials greater than two. Hence,

$\frac{d^{2} \varphi(z)}{d z}+\frac{(1-z)}{z(1-z)} \frac{d \varphi(z)}{d z}+\frac{1}{z^{2}(1-z)^{2}}\left[\begin{array}{l}\frac{2 \mu E_{n j}}{\zeta^{2} \hbar^{2}}-\frac{6 \mu e^{2}}{\zeta^{2} \hbar^{2} \pi \varepsilon_{0} R_{c}}+\frac{2 \mu e^{2}}{\zeta^{4} \hbar^{2} \pi \varepsilon_{0} R_{c}^{3}}- \\ l(l+1)+\frac{12 \mu e^{2} z}{\zeta^{2} \hbar^{2} \pi \varepsilon_{0} R_{c}}-\frac{4 \mu E_{n j} z}{\zeta^{2} \hbar^{2}}+\frac{2 \mu \eta z}{\zeta \hbar^{2}} \\ +\frac{2 \mu \eta_{(s o)} r_{0}^{2} \zeta}{\hbar^{2}}\left(j(j+1)-l(l+1)-\frac{3}{4}\right) z \\ -\frac{8 \mu e^{2} z}{\zeta^{4} \hbar^{2} \pi \varepsilon_{0} R_{c}^{3}}+\frac{2 \mu E_{n j} z^{2}}{\zeta^{2} \hbar^{2}}-\frac{2 \mu \eta z^{2}}{\zeta \hbar^{2}} \\ +\frac{\mu \eta_{(s o)} r_{0}^{2} \zeta}{\hbar^{2}}\left(j(j+1)-l(l+1)-\frac{3}{4}\right) z^{2} \\ -\frac{6 \mu e^{2} z^{2}}{\zeta^{2} \hbar^{2} \pi \varepsilon_{0} R_{c}}+\frac{12 \mu e^{2} z^{2}}{\zeta^{4} \hbar^{2} \pi \varepsilon_{0} R_{c}^{3}}\end{array}\right] \varphi(z)=0$,

where,

$-\chi^{2}=\frac{2 \mu E_{n l j}}{\zeta^{2} \hbar^{2}}-\frac{6 \mu e^{2}}{\zeta^{2} \hbar^{2} \pi \varepsilon_{0} R_{c}}+\frac{2 \mu e^{2}}{\zeta^{4} \hbar^{2} \pi \varepsilon_{0} R_{c}^{3}}-l(l+1)$

$v=\frac{12 \mu e^{2}}{\zeta^{2} \hbar^{2} \pi \varepsilon_{0} R_{c}}-\frac{4 \mu E_{n l j}}{\zeta^{2} \hbar^{2}}+\frac{2 \mu \eta}{\zeta \hbar^{2}}+\frac{2 \mu \eta_{(s o)} r_{0}^{2} \zeta}{\hbar^{2}}$

$\left(j(j+1)-l(l+1)-\frac{3}{4}\right)-\frac{8 \mu e^{2}}{\zeta^{4} \hbar^{2} \pi \varepsilon_{0} R_{c}^{3}}$

$\frac{d^{2} \varphi(r)}{d r^{2}}+\frac{2 \mu}{\hbar^{2}}\left[\begin{array}{l}E_{n l j}+\frac{\eta e^{-\alpha r}}{r}+\frac{1}{2} \eta_{(s o)} r_{0}^{2} \frac{1}{r}\left(\zeta \frac{e^{-\zeta r}}{r}+\frac{e^{-\zeta r}}{r^{2}}\right)\left(j(j+1)-l(l+1)-\frac{3}{4}\right) \\ -\left(\frac{3 e^{2}}{\pi \varepsilon_{0} R_{c}}-\frac{e^{2} r^{2}}{\pi \varepsilon_{0} R_{c}^{3}}\right)-\frac{\hbar^{2}}{2 \mu r^{2}} l(l+1)\end{array}\right] \begin{gathered}-\delta=\frac{2 \mu E_{n l j}}{\zeta^{2} \hbar^{2}}-\frac{2 \mu \eta}{\zeta \hbar^{2}}+\frac{\mu \eta_{(s o)} r_{0}^{2} \zeta}{\hbar^{2}} \\ (j)=0 \\ \left(j(j+1)-l(l+1)-\frac{3}{4}\right)-\frac{6 \mu e^{2}}{\zeta^{2} \hbar^{2} \pi \varepsilon_{0} R_{c}}+\frac{12 \mu e^{2}}{\zeta^{4} \hbar^{2} \pi \varepsilon_{0} R_{c}^{3}}\end{gathered}$

To get the solution of (18), we need another change of variable with:

$z=e^{-\zeta r}$

To deal with the centrifugal barrier, we introduce the Greene-Aldrich approximation scheme [45]-[47] to analytically solve the equation. This approximation scheme is an excellent approximation to the centrifugal term, which is valid for $\zeta<<1$. It has the form
By substituting (23), (24) and (25) into (22) we have:

$$
\begin{aligned}
& \frac{d^{2} \varphi_{n l j}(z)}{d z}+\frac{(1-z)}{z(1-z)} \frac{d \varphi_{n l j}(z)}{d z}+ \\
& \frac{1}{z^{2}(1-z)^{2}}\left[-\delta z^{2}+v z-\chi^{2}\right] \varphi_{n l j}(z)=0 .
\end{aligned}
$$

After comparing (26) with (2), we have the appropriate polynomial as:

$$
\begin{aligned}
& \tilde{\tau}(z)=1-z, \quad \sigma(z)=z(1-z), \\
& \sigma^{\prime}(z)=1-2 z \quad \sigma^{\prime \prime}(z)=-2, \quad \tilde{\sigma}(z)=-\delta z+v z-\chi^{2} .
\end{aligned}
$$


Upon inserting the polynomials given by (27) into (10) gives the polynomial:

$\pi(z)=-\frac{z}{2} \pm \sqrt{(\Upsilon-k) z^{2}+(k-v) z+\chi^{2}}$

where

$\Upsilon=\frac{1}{4}+\delta$

Based on the NU method, the quadratic form under the square root sign of (28) must be solved if we set the discriminant of this quadratic equation equal to zero: $\Delta=b^{2}-4 a c=0$. This discriminant gives a new quadratic equation, which can be solved for the constant $k$ to get the two roots. Here, we take the negative root given as

$K=v-2 \chi\left[\chi-\sqrt{\Upsilon-v+\chi^{2}}\right]$

$\tau(z)$ given in (6) has a negative derivative expression for this value of $\pi(z)$ [18] using (30), the most suitable expression of $\pi(z)$ is chosen as:

$\pi(z)=-\frac{z}{2}+\chi-z\left[\chi+\sqrt{\Upsilon-v+\chi^{2}}\right]$

Hence, $\tau(z)$ and $\tau^{\prime}(z)$ are obtain as:

$\tau(z)=1+2 \chi-2 z\left[1+\chi+\sqrt{\Upsilon-v+\chi^{2}}\right]$

$\tau^{\prime}(z)=-2-2 \chi-2 \sqrt{\Upsilon-v+\chi^{2}}$

using (27), (31) and (32), the following expressions for $\lambda$ and $\lambda_{n}$ are obtained explicitly:

$$
\begin{aligned}
& \lambda=-\frac{1}{2}-\sqrt{\Upsilon-v+\chi^{2}}+v-\chi\left[1-2 \chi-2 \sqrt{\Upsilon-v+\chi^{2}}\right] \\
& \lambda_{n}=n^{2}+n+2 n\left[\chi+\sqrt{\Upsilon-v+\chi^{2}}\right]
\end{aligned}
$$

By comparing (33) and (34), we obtain the dimensionless parameter $\chi$ as:

$$
\chi=-\frac{1}{2}\left[\frac{\left(n+\frac{1}{2}\right)^{2}+2\left(n+\frac{1}{2}\right) \sqrt{\frac{1}{4}+\delta-v+\chi^{2}}+\frac{1}{4}+2 \chi^{2}-v}{\left(n+\frac{1}{2}+\sqrt{\frac{1}{4}+\delta-v+\chi^{2}}\right)}\right]
$$

With the help of (23)-(25), we obtain bound state energy eigenvalues of Yukawa potential plus spin-orbit coupling and Coulomb interaction term as a function of quantum numbers $n, l$, and $j$ as:

$$
\begin{aligned}
& E_{n l j}=\frac{\hbar^{2} \zeta^{2} l(l+1)}{2 \mu}+\frac{3 \mu e^{2}}{\pi \varepsilon_{0} R_{c}}-\frac{e^{2}}{\zeta^{2} \pi \varepsilon_{0} R_{c}^{3}}-\frac{\hbar^{2} \zeta^{2}}{8 \mu} \times \\
& {\left[\begin{array}{l}
\left(n+\frac{1}{2}\right)^{2}+2\left(n+\frac{1}{2}\right) \sqrt{\frac{1}{4}+l(l+1)-\frac{3 \mu \eta_{(s o)} r_{o}^{2} \zeta\left(j(j+1)-l(l+1)-\frac{3}{4}\right)}{\hbar^{2}}-\frac{6 \mu e^{2}}{\zeta^{4} \hbar^{2} \pi \varepsilon_{0} R_{c}^{3}}} \\
+\frac{1}{4}+2 l(l+1)-\frac{2 \mu \eta}{\zeta h^{2}}-\frac{2 \mu \eta_{(s o)} r_{o}^{2} \zeta\left(j(j+1)-l(l+1)-\frac{3}{4}\right)}{\hbar^{2}}+\frac{4 \mu e^{2}}{\zeta^{4} \hbar^{2} \pi \varepsilon_{0} R_{c}^{3}} \\
\left(n+\frac{1}{2}+\sqrt{\frac{1}{4}+l(l+1)-\frac{3 \mu \eta_{(s o)} r_{o}^{2} \zeta\left(j(j+1)-l(l+1)-\frac{3}{4}\right)}{\hbar^{2}}-\frac{6 \mu e^{2}}{\zeta^{4} \hbar^{2} \pi \varepsilon_{0} R_{c}^{3}}}\right)
\end{array}\right.}
\end{aligned}
$$

Next is to find the wave function. Using (9), the weight function $\rho(z)$ is of the form:

$$
\rho(z)=z^{2 \chi}(1-z)^{2 \sqrt{\frac{1}{4}+\delta-v+\chi^{2}}}
$$

and when (37) is used in (8), the Rodrigues relation is found:

$$
\begin{aligned}
& y_{n}=B_{n} z^{-2 \chi}(1-z)^{-2 \sqrt{\frac{1}{4}+\gamma-\beta+\chi^{2}}} \frac{d^{n}}{d z^{n}}\left[z^{n+2 \chi}(1-z)^{n+2 \sqrt{\frac{1}{4}+\gamma-\beta+\chi^{2}}}\right] \\
& \left.\equiv P_{n}^{\left(2 \varepsilon, 2 \sqrt{\frac{1}{4}+\gamma-\beta+\chi^{2}}\right.}\right)(1-z),
\end{aligned}
$$

where $P_{n}$ is the Jacobi polynomial. From Eq. (5):

$$
\phi(z)=v^{\chi}(1-z)^{\left(\frac{1}{2}+\sqrt{\frac{1}{4}+\delta-v+\chi^{2}}\right)}
$$

is obtained and the wavefunction becomes:

$\psi_{n l}(z)=\mathrm{N}_{n l} z^{\chi}(1-z)^{\left(\frac{1}{2}+\sqrt{\frac{1}{4}+\delta-v+\chi^{2}}\right)} P_{n}^{\left(2 \chi, \frac{1}{2}+\sqrt{\frac{1}{4}+\delta-v+\chi^{2}}\right)}(1-2 z)$

Where $N_{n l}$ is the normalization constant. Using the normalization condition, we obtain the normalization constant as follows:

$\frac{\mathrm{N}_{n l}^{2}}{\zeta} \int_{-1}^{1}\left(\frac{1-\gamma}{2}\right)^{2 \chi}\left(\frac{1+\gamma}{2}\right)^{\beta}\left[P_{n}^{(2 \chi, 2 \beta-1)}(\gamma)\right]^{2} d \gamma=1$

Equation (41) is obtained by setting:

$$
\left.\begin{array}{l}
\beta=1+\sqrt{1+4 \delta-4 v+4 \chi^{2}}, \\
\beta-1=\sqrt{1+4 \delta-4 v+4 \chi^{2}}
\end{array}\right\}
$$

Hence by comparing (41) with standard integral of the form of (37) of [59]:

$$
\begin{aligned}
& \int_{-1}^{1}\left(\frac{1-p}{2}\right)^{x}\left(\frac{1+y}{2}\right)^{y}\left(P_{n}^{(2 x, 2 y-1)}(p)\right)^{2} d p \\
& =\frac{2 \Gamma(x+n+1) \Gamma(y+n+1)}{n ! x \Gamma(x+y+n+1)}
\end{aligned}
$$

we obtain the normalization constant as: 


$$
\mathrm{N}_{n l j}=\left[\frac{n ! \chi \zeta \Gamma(2 \chi+\beta+n+1)}{\Gamma(2 \chi+n+1) \Gamma(\beta+n+1)}\right]^{\frac{1}{2}}
$$

\section{SPECIAL CASES}

However, no experimental evidence for the solution of (36) has been recorded to the best of our knowledge. Therefore, our calculations are only for academic purposes; hence, the need to test for the accuracy of our calculation. To do this, we consider the following cases.

\section{A. Case 1}

If we set $R_{c}=0$ in (17), (36) yield energy eigenvalue of Yukawa potential with spin-orbit coupling term:

$$
\left.\begin{array}{rl}
E_{n l j} & =\frac{\hbar^{2} \zeta^{2} l(l+1)}{2 \mu}- \\
& \frac{\hbar^{2} \zeta^{2}}{8 \mu}\left[\frac{\left(n+\frac{1}{2}\right)^{2}+2\left(n+\frac{1}{2}\right) \sqrt{\frac{1}{4}+l(l+1)-\frac{3 \mu \eta_{(s o)} r_{o}^{2} \zeta\left(j(j+1)-l(l+1)-\frac{3}{4}\right)}{\hbar^{2}}}}{\left(n+\frac{1}{2}+\sqrt{\left.\frac{1}{4}+l(l+1)-\frac{3 \mu \eta_{(s o)} r_{o}^{2} \zeta\left(j(j+1)-l(l+1)-\frac{3}{4}\right)}{\hbar^{2}}\right)}\right)}\right.
\end{array}\right]
$$

Equation (45) is similar to (27) of [35].

\section{B. Case 2}

If we set $\eta_{s 0}=R_{c}=0$ in (17), we have energy eigenvalue of Yukawa potential as:

$$
E_{n l j}=\frac{\hbar^{2} \zeta^{2} l(l+1)}{2 \mu}-\frac{\hbar^{2} \zeta^{2}}{8 \mu}\left[\frac{(n+l+1)^{2}+l(l+1)-\frac{2 \mu \eta}{\zeta \hbar^{2}}}{(n+l+1)}\right]^{2}
$$

Equation (46) is similar to (34) of [49]; (19) of [50]; (2) of [51]; (52) of [52]; (75) of [53]; and (27) of [54]

\section{DISCUSSION}

Table 1 shows the numerical single-particle energy spectrum of the doubly magic nuclide ${ }_{28}^{56} \mathrm{Ni}(\mathrm{Z}=28, \mathrm{~N}=$ $28)$, and a single magic nuclide ${ }_{50}^{116} \mathrm{Sn}(\mathrm{Z}=50, \mathrm{~N}=66)$ respectively. For the spin-orbit interaction, the spin orientation degeneracy is removed in the main spin-orbit levels, causing splitting of energy levels. By Including total angular momentum quantum number $j$, the numerical energy spectrum was obtained at $1 p_{\frac{1}{2}}, 1 d_{3 / 2}$ and $2 s_{\frac{1}{2}}$ for ${ }_{28}^{56} \mathrm{Ni}$ and $1 p_{\frac{1}{2}}, 1 d_{3 / 2}, 2 s_{1 / 2}, 1 f_{5 / 2}$ and $2 p_{1 / 2}$ for ${ }_{50}^{116} S n$, respectively. We observed that the larger the orbital angular momentum quantum numbers $\ell$, the larger the splitting. For $\ell=0$, only $j=\frac{1}{2}$ is possible, hence no splitting and we have the ground state of the two magic nuclei. This is indicated by $2 s_{1 / 2}$ state.

To impose the electromagnetic interaction on the singleproton levels, one may assume that each proton lies in the repulsive Coulomb potential well, made by the other protons. To correct single-proton levels, we understood that single point-like proton lies in a uniformly charged spherical potential well with a radius. In contrast, these corrections do not lead to a significant change in the energy levels of the nuclei [55]. However, the energy distributions of nucleons are affected by the strong short-range correlations of nucleons in finite nuclei. It becomes essential when we calculate the nucleon single-particle energies and the choice of the potential parameters. Brown [56] suggested that one could introduce up to six parameters for the spin-orbit interaction for the best fit of nuclear single-particle energies. Unfortunately, the available experimental information on the single-particle energies of the deep bound states is scanty [57].

TABLE 1: SiNGLE-ENERGY SPECTRUM OF ${ }^{56} \mathrm{Ni}$ AND ${ }^{116} \mathrm{Sn}$ NUCLIDES FoR: $R=r_{0} A^{1 / 3}, \eta=40+0.13 A, \quad \zeta=1.54 \mathrm{fm}, h=6.582850502 \times 10^{-22} \mathrm{MeV} . s$,

\begin{tabular}{ccccc}
\multicolumn{5}{c}{$e=1.19999(\mathrm{MeV} . f m)^{1 / 2}, \eta_{(s 0)}=0.44 \eta$} \\
\hline Nuclide & $j$ & $J^{\pi}$ & $\eta(\mathrm{MeV})$ & $E_{n j}(\mathrm{MeV})$ \\
\hline \multirow{3}{*}{${ }^{56} \mathrm{Ni}$} & $1 p_{1 / 2}$ & $0^{+}$ & 47.28 & -40.204732939 \\
& $1 d_{3 / 2}$ & & & -62.01202362 \\
& $2 s_{1 / 2}$ & & & -127.4261691 \\
\hline \multirow{6}{*}{${ }^{116} \mathrm{Sn}$} & $1 p_{1 / 2}$ & $0^{+}$ & 55.08 & -75.2 .9801832 \\
& $1 d_{3 / 2}$ & & & -114.2349840 \\
& $2 s_{1 / 2}$ & & & -231.0459655 \\
& $1 f_{5 / 2}$ & & & -153.1719709 \\
& $2 p_{1 / 2}$ & & & -464.6679917 \\
\hline
\end{tabular}

\section{CONCLUSION}

In this study, we obtained the approximate analytical solutions of the Schrödinger equation for the Yukawa potential plus spin-orbit and Coulomb interaction terms by approximation to the centrifugal barrier using the NU method. Explicitly, we have computed the single-energy spectrum at different states of the two magic nuclei using energy eigenvalues obtained. We have also obtained the corresponding normalized wave functions for the magic nuclei presented in terms of Jacobi polynomial. Therefore, the proposed model can also be used to investigate other magic nuclei.

\section{ACKNOWLEDGMENT}

E. S. William would like to thank Dr. A. N. Ikot from the Department of Physics, University of Port Harcourt for his encouragement for the successful completion of this work. 


\section{REFERENCES}

[1] S. M. Lenzi and M. A. Bentley, "Isobaric analogue states studied in mirrored fragmentation and knockout reactions," Mod. phys lett A, 1891-1894 (2010).

[2] E. B. Suckling, "Nuclear Structure and Dynamics from the Fully Unrestricted Skyrme-Hartree-Fock Model," Ph. D thesis, Department of Physics, University of Surrey, 2011.

[3] V. F. Robert and R. V. F. Janssens, Elusive magic number, Nature 435, 897-898 (2005).

[4] K. S. Krane, Introductory Nuclear Physics, New York, $3^{\text {rd }}$ edn, John Wiley \& sons Inc, D Hallidays, 1988.

[5] M. Goeppert-Meyer, On Closed Shells in Nuclei, Phys. Rev. 75 (1949).

[6] M. Goeppert-Mayer, and J. Jensen, Elementary theory of nuclear shell structure, New York, Wiley, 224, (1955)

[7] V. G. Solov'ev, Theory of atomic nuclei, England, Inst. Phys, 33, (1992).

[8] P. Ring, and P Schuck, The nuclear many-body problem, New York, Springer-Verlag, 42, (1980).

[9] H. Heylen, Ground state properties near the $N=20$ and $N=40$ islands of inversion, $\mathrm{PhD}$ dissertation, University of Liverpool, $\mathrm{Ku}$ Leuven, Arenberg Doctorial school, (2016).

[10] R. V. F. Janssens, Unexpected doubly magic nucleus, Nature news and views 459 (2009)

[11] S. G. Nilsson and I. Ragnarsson, Shapes and Shells in Nuclear Structure, Cambridge, Cambridge University Press, 100, (1995).

[12] R. D. Rietz, Effective Charges Near ${ }^{56} \mathrm{Ni}$ and production of antinuclei studied with heavy-ion reactions $\mathrm{A} \mathrm{Ph}$. D thesis, USA Department of Physics Lund University, (2005).

[13] R. C. Cockrell, Ab Initio Nuclear Structure Calculations for Light Nuclei Graduate Theses, Iowa State University, 12654 (2012).

[14] A. Belyaev, Nucl. Part. Phys. S 3002, 1-163 (2015).

[15] K. M. Hanna, S. H. M. Sewailem, R. Hussien, L. I. Abou-Salem, and A. G. Shalaby, The Ground-State Calculations for Some Nuclei by Mesonic Potential of Nucleon-Nucleon Interaction. Adv. High Energy Phys, 1-15 (2020).

[16] S. Bogner, T. T. S. Kuo, L. Coraggio, A. Covello, and N. Itaco, Low momentum nucleon-nucleon potential and shell model effective interactions, Phys. Rev. C 65, 5, 1-5 (2002).

[17] B. B. Sahu, S. K. Singh, M. Bhuyan, S. K. Biswal, and S. K. Patra, Importance of nonlinearity in the NN potential, Phys. Rev. C 89, 1-8 034614 (2014)

[18] A. F. Nikiforov and V. B. Uvarov, Special Functions of Mathematical Physics, Birkhauser, Basel, (1988).

[19] C. O. Edet, U. S. Okorie, A. T. Ngiangia, A. T. Ngiangia and A. N. Ikot, "Bound state solutions of the Schrodinger equation for the modified Kratzer potential plus screened Coulomb potential," Indian J Phys, 94, 425-433 (2019).

[20] E. S. William, E. P. Inyang, E. A. Thompson, Arbitrary $\ell$-solutions of the Schrödinger equation interacting with Hulthén-Hellmann potential model, Rev Mex Fis, 66,6, 1-11 (2020)

[21] E. P. Inyang et al. Any State Solutions of the Schrödinger equation interacting with class Of Yukawa - Eckart potentials. Intl J. Innov Sci, Engr. Tech.7, 11 (2020).

[22] E. P. Inyang, E. P. Inyang, I. O. Akpan, J. E. Ntibi and E. S. Willam, Analytical solutions of the Schrodinger equation with class of Yukawa potential for a quarkonium system via series expansion method, European J. of Applied Phys. (2020).

[23] C. A. Onate, and M. C. Onyeaju, Dirac particles in the field of Frost-Musulin diatomic potential and the thermodynamic properties via parametric Nikiforov-Uvarov method, Sri. J. Phys.17, 1-17 (2016).

[24] E. P. Inyang, E. P. Inyang, E. S. William and E. E. Ibekwe, Study on the applicability of Varshn potential to predict the mass spectra of quark-antiquark systems in non-relativistic framework, Jord. J. Phys. (2020) JJ14-11-10-020.

[25] K. J. Oyewumi, B. J. Falaye, C. A. Onate, O. J. Oluwadare and W. A. Yahya, Thermodynamic properties and the approximate solutions of the Schrödinger equation with the shifted Deng-Fan potential model, Mol. Phys. 112, 127-141 (2014).

[26] Bayrak, and I. Boztosun, Bound state solutions of the Hulthén potential by using the asymptotic iteration method, Phys. Scr. 76, 1 , 92-96 (2007).

[27] E Ateser, H Ciftci, M U gurlu Chin. J. Phys. 45 (2007).

[28] M. Aygun, O. Bayrak, and I. Boztosun, Journal of Physics B: Atomic, Molecular and Optical Physics solution of the radial Schrödinger equation for the potential family $\mathrm{V}(\mathrm{r})=\mathrm{A} / \mathrm{r}^{2}-\mathrm{B} / \mathrm{r}+\mathrm{Cr}^{\mathrm{k}}$ using the asymptotic iteration method, J. Phys. B Mol. Opt. Phys. 40, 537-544 (2007)

[29] H. Hassanabadi, S. Zarrinkamar, B. H. Yazarloo, Spectrum of a Hyperbolic Potential via SUSYQM within the Semi-Relativistic Formalism, Chin. J. Phys. 50,5 1-7 (2012)

[30] A. N. Ikot, H. P. Obong, H. Hassanabadi, Relativistic Symmetries of $(\mathrm{D}+1 \mathrm{D}+1)$ Dimensional Dirac Equation with Multiparameter Exponential-Type Potentials Using Supersymmetric Quantum Mechanics, Few-Body Syst. 56,185-196 (2015).

[31] C. A. Onate and J. O. Ojonubah, Relativistic and non-relativistic solutions of the generalized Poschl-Teller and Hyperbolic potentials, Intl. J. Mod.Phys. E, 24, 1-16 (2015)

[32] S. H. Dong, Factorization Method in Quantum Mechanics, Brelin, Springer, 1-295 (2007)

[33] C. O. Edet, and P. O. Okoi, Any $l$-state solutions of the Schrödinger equation for $q$-deformed Hulthen plus generalized inverse quadratic Yukawa potential in arbitrary dimensions Rev. Mex. de Fis. 65 (2019).

[34] S. H. Dong, Wave Equation in Higher Dimensions, Berlin, 1-299 (Springer, 2011).

[35] M. R. Pahlavani and S A. Alavi, Solutions of Woods-Saxoon potential with spin-orbit and centrifugal terms through NikiforovUvarov method, Comm. Theor. Phys, 58, 5, 739-743 (2012).

[36] A. A. Ribha, Nuclear Physics; Chapter 2: Binding Energy \& Nuclear Models, Al-Mustansiriyah University, College of Science Physics Department, 1-29 (2016).

[37] T. Otsuka, R. Fujimoto, Y. Utsuno et al. Magic Numbers in Exotic Nuclei and Spin-Isospin Properties of the NN Interaction, Phys. Rev. Lett. 87, 8. 1-4 (2001)

[38] R. Gupta and S. S. Malik Rev. Mexi. de Fis. 661 (2020),

[39] H. Yukawa, On the interaction of elementary particles, Proc. Phys. Math. Soc. 17 48, (1935).

[40] J. C. Del Valle, and D. J. Nader, Radial anharmonic oscillator: Perturbation theory, new semiclassical expansion, approximating eigenfunctions. I. Generalities, cubic anharmonicity case, Intl. J. Mod. Phys. 5, 1-43 (2018).

[41] Y. Li, X. Luo, and H. Kröger, Bound states and critical behavior of the Yukawa potential. Sci China Ser G 49, 60-71 (2006).

[42] M. Hamzavi, K. E. Thylwe, and A. A. Rajabi, Approximate Bound States Solution of the Hellmann Potential, Comm. Theor. Phys. 60,1, 1-8 (2013)

[43] M. Falek, N. Belghar, and M. Moumni, Exact solution of Schrödinger equation in (anti-)de Sitter spaces for hydrogen atom, Eur. Phys. J. Plus 135 335, 1-12 (2020).

[44] A. Niknam, A. A. Rajabi, M. Solaimani, Solutions of D dimensional Schrodinger equation for Woods-Saxon potential with spin-orbit, coulomb and centrifugal terms through a new hybrid numerical fitting Nikiforov-Uvarov method J. Theor. Appl. Phys. $10,53-59$ (2016)

[45] R. L. Greene and C. Aldrich, R. L. Greene and C. Aldrich Phys Rev. A 14, 2363 (1976) Phys. Rev. A 14, 2363 (1976).

[46] S. H. Dong, W. C. Qiang, G. H. Sun, V. R. Bezerra, J. Phys A Math. Theo, Analytical approximations to the $l$-wave solutions of the Schrödinger equation with the Eckart potential, J. Phys. A 40, 1 7 (2007)

[47] Ebomwonyi, C. A. Onate, M. C. Onyeaju, A. N. Ikot, Any l-states solutions of the Schrodinger equation interacting with Hellmanngeneralized Morse potential model Karbala Intl. J. Mod. Sci 3, 1-10 (2017)

[48] A. Tas, O. Aydogdu, and M. Salti, Relativistic spinless particles with position dependent mass: Bound states and scattering phase shift, J. Korean Phys. Soci, 70, 896-904 (2017).

[49] A. D. Antia, E. E. Umo, C. C. Umoren, Solutions of nonrelativistic Schrödinger equation with Hulthen-Yukawa plus angle dependent potential within the framework of Nikiforov-Uvarov method, $J$. Theor. Phys. Crypt 10, 1-8, (2015).

[50] M. Hamzavi, M. Movahedi, K. E. Thylwe, and A. A. Rajabi, Approximate Analytical Solution of the Yukawa Potential with Arbitrary Angular Momenta, Chinese Phys. Lett. 29,8, 1-5, (2012).

[51] E. Z. Liverts et al, Analytic presentation of a solution of the Schrodinger equation, Few-body syst, 44, 367-370 (2008).

[52] S. M. Ikhdair, Approximatek-state solutions to the Dirac-Yukawa problem based on the spin and pseudospin symmetry, 10(2), 361381 (2012)

[53] B. I. Ita et al. Bound State Solutions of the Schrödinger Equation for the More General Exponential Screened Coulomb Potential Plus Yukawa (MGESCY) Potential Using Nikiforov-Uvarov Method, $J$. Quantum Inform. Sci. 8, 1, 24-45 (2018). 
[54] C. A. Onate et al. Approximate eigensolutions of the attractive potential via parametric Nikiforov-Uvarov method, Heliyon 4 (2018).

[55] H. Mariji, The Calculation of Single-Nucleon Energies of Nuclei by Considering Two-Body Effective Interaction, and a Hartree-Fock Inspired Scheme, Adv. High Energy Phys. 2398198 (2016), 1-10.

[56] B. A. Brown, Lecture Notes in Nuclear Structure Physics 1, National Superconducting Cyclotron Laboratory, Michigan State University, Dept of Physics and Astronomy, E. Lansing, MI 48824 (2011).

[57] S. Fujii, R. Okamoto, and K. Suzuki, Ground-State and SingleParticle Energies of Nuclei around ${ }^{16} \mathrm{O},{ }^{40} \mathrm{Ca}$, and ${ }^{56} \mathrm{Ni}$ from Realistic Nucleon-Nucleon Forces Phys. Rev. Lett. 103, 182501 (2009).

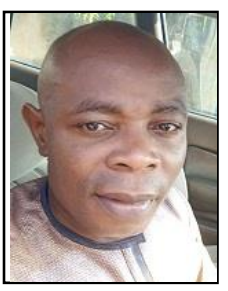

E. S. William was born in Akwa Ibom State on the 30th April. He holds a Ph. D in Theoretical Physics, M.Sc. in Nuclear Physics, and B.Sc. in Physics all from the University of Calabar, Calabar, Cross River State, Nigeria.

He is currently a Physics teacher in Nigerian Navy Secondary School, Calabar and a research Assistant in the Department of Physics, University of Calabar, Calabar Nigeria. He has published in reputable journals of Physics.

1. E. S. William, E. P. Inyang, E. A. Thompson, Arbitrary $\ell$-solutions of the Schrödinger equation interacting with Hulthén-Hellmann potential model, Rev Mex Fis, 66,6, 1-11 (2020).

2. E. P. Inyang, E. S. William and J. A. Obu, "Eigensolutions of the Ndimensional Schrödinger equation interacting with Varshni-Hulthen potential" Revista Mexicana de Fisica.66 2020.

3. P. Inyang, E. P. Inyang, E. S. William, and E. E. Ibekwe, "Study on the applicability of Varshni potential to predict the mass-spectra of the Quark-antiquark systems in a non-relativistic framework", Jordan Journal of Physics, 14-11-10-020,2020.

Dr. William is currently working on symmetry breaking in mirror nuclei. 